\title{
The Role of Body Mass Index on Perioperative Medical Complications and Outcomes Following Primary Total Knee Arthroplasty \\ Ashton Bosler, ${ }^{1}$ Evan R. Deckard, ${ }^{2}$ Mary Ziemba-Davis, ${ }^{2}$ \\ Leonard T. Buller, ${ }^{2}$ R. Michael Meneghini ${ }^{2}$
}

${ }^{1}$ Indiana University School of Medicine; ${ }^{2}$ Indiana University School of Medicine, Department of Orthopaedic Surgery

Background and Hypothesis: Obesity is associated with increased medical complications and septic revision following total knee arthroplasty (TKA); however, evidence-based multidisciplinary perioperative care coordination can optimize outcomes. This study examined the relative influence of body mass index (BMI) on outcomes following TKA in an arthroplasty program with specialized perioperative medical care.

Experimental Design or Project Methods: 284 consecutive primary unilateral TKAs performed by one surgeon at a designated hip and knee center between 2019 and 2020 were retrospectively reviewed. Outcomes including (1) inpatient and 90-day medical complications (cardiac, renal, respiratory, venous thromboembolism, cerebrovascular accident, joint infection, sepsis, and death), (2) 90-day emergency department (ED) visits and hospital readmissions, and (3) 90-day reoperations on the index joint were evaluated in relation to BMI.

Results: $59.9 \%$ of the sample was non-obese (BMI $<35 \mathrm{~kg} / \mathrm{m}^{2}$ ), $20.1 \%$ severely obese ( $\geq 35$ $\left.\mathrm{kg} / \mathrm{m}^{2}\right), 18.3 \%$ morbidly obese $\left(\geq 40 \mathrm{~kg} / \mathrm{m}^{2}\right)$, and $1.8 \%$ super-obese $\left(\geq 50 \mathrm{~kg} / \mathrm{m}^{2}\right)$. There were five inpatient complications (40\% non-obese, $60 \%$ morbidly obese patients, $p=1.000)$. $64 \%$ of 1190 -day ED visits were made by non-obese patients and $36 \%$ were made by severely/morbidly obese patients $(p=0.395)$. Nine hospital readmissions within 90 -days represented $67 \%$ non-obese and $33 \%$ severely/morbidly obese patients $(p=0.395)$. All complications were related to renal/respiratory/cardiac issues or joint infection. There were five reoperations of the index joint within 90 days (40\% non-obese, $60 \%$ severely/morbidly obese, $p=1.000$ ), with infection or arthrofibrosis as the underlying cause.

Conclusion and Potential Impact: We observed a similar distribution of perioperative medical complications following primary TKA regardless of BMI. Further evaluation with a larger sample and using machine learning to control for the influence of multiple potential confounders will help determine the relative contribution of various comorbidities associated with obesity on outcomes following TKA. An awareness of the relative contribution of BMI on these outcomes may improve preoperative counseling, management, and treatment of obese patients who undergo TKA. 